\title{
A Vision of Pharmacy Practice Excellence
}

\author{
Patricia Macgregor
}

$\mathrm{I}$ n 2008, the Canadian Society of Hospital Pharmacists (CSHP) launched its CSHP 2015 vision of pharmacy practice excellence, challenging its members nationwide to commit to enhancing pharmacy practice for improved patient care and medication-related outcomes. Today, entering the final 18 months of this patient-centred initiative, we can proudly report on the many actions undertaken on the 36 evidencebased objectives within the 6 key goals of CSHP 2015.

Hospital pharmacy leaders were asked to commit to supporting their pharmacists' role in implementing CSHP 2015 and to sharing accomplishments with colleagues across the country. The shared expertise of members from every CSHP branch has generated an incredible number of resources, including 30 success stories, 34 virtual posters, 3 toolkits, and 10 archived webinars. All resources are posted on the CSHP website (www.cshp.ca/cshp2015). Pharmacists have reported the usefulness of the toolkits during implementation of new processes. In addition, the high attendance at CSHP webinars is a testament to the value that the Society brings from this form of sharing expertise, and the reach of knowledge dissemination is demonstrated by communications received from colleagues across the globe. In addition to website resources, recent opportunities for dissemination of knowledge about CSHP 2015 included the Professional Practice Conference (PPC) and the Harrison Pharmacy Management Seminar.

In June 2013, hospital pharmacy directors were surveyed to determine their CSHP 2015 needs. The survey results, shared during PPC 2014, identified 10 key CSHP 2015 objectives for which directors would like support; of these, the top 3 are organization-wide programs to review safe medication use, management and monitoring of inpatients' medication therapy by pharmacists, and bar-coding technology. The results also highlighted other priorities that compete for pharmacy directors' time.

Measurement of key performance indicators is an important aspect of determining value. The tools developed for CSHP
2015 offer an excellent opportunity for pharmacists to share progress on their journey to excellence, to highlight and celebrate successes, and to educate others about the value proposition of pharmacists in medication-related outcomes. Also highlighted at PPC were the CSHP 2015 progress charts, facilitated

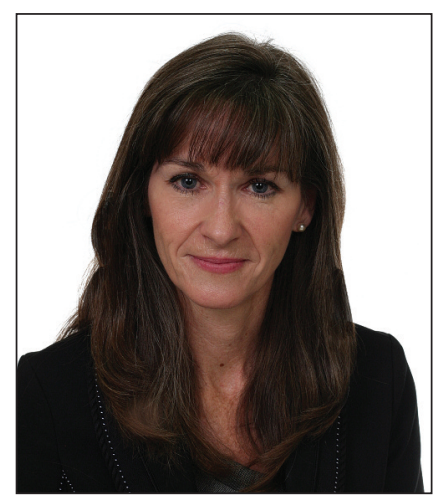
through data collection by the Hospital Pharmacy in Canada survey. These progress charts provide convenient dashboards to compare the progress of a hospital pharmacy department against regional achievement of the CSHP 2015 objectives. The Hospital Pharmacy in Canada survey is conducted biennially, and the upcoming survey (spring 2014) will therefore be the last opportunity to measure progress on CSHP 2015 objectives through this channel.

In preparation for the culmination of CSHP 2015, CSHP is creating a video collage, initiated at this year's PPC, to highlight pharmacists' stories about how CSHP 2015 has affected pharmacy practice and patient care.

As we draw toward the final stages of CSHP 2015, I encourage the pharmacy community and individual pharmacists to further embrace this vision of practice excellence, to attend the PPC in February 2015 for the festive celebration of CSHP 2015 's finale, and to continue working toward improved patient care and medication-related outcomes by advancing pharmacy practice.

Patricia Macgregor, BSC, RPh, MRPharmS, MHSC, CHE, is President and Vision Liaison for the Canadian Society of Hospital Pharmacists. 